Draft Version OCtober 27, 2018

Preprint typeset using $\mathrm{IAT}_{\mathrm{E}} \mathrm{X}$ style emulateapj v. 5/2/11

\title{
SEEING THE FIRST SUPERNOVAE AT THE EDGE OF THE UNIVERSE WITH JWST
}

\author{
Daniel J. Whalen ${ }^{1}$, Chris L. Fryer ${ }^{2}$, Daniel E. Holz ${ }^{3}$, Alexander Heger ${ }^{4}$, S. E. Woosley ${ }^{5}$, Massimo Stiavelli ${ }^{6}$, \\ Wesley Even ${ }^{7}$ AND LuCille H. FreY ${ }^{7,8}$ \\ Draft version October 27, 2018
}

\begin{abstract}
The first stars ended the cosmic Dark Ages and created the first heavy elements necessary for the formation of planets and life. The properties of these stars remain uncertain, and it may be decades before individual Pop III stars are directly observed. Their masses, however, can be inferred from their supernova explosions, which may soon be found in both deep-field surveys by the James Webb Space Telescope (JWST) and in all-sky surveys by the Wide Field Infrared Survey Telescope (WFIRST). We have performed radiation hydrodynamical simulations of the near infrared signals of Pop III pairinstability supernovae in realistic circumstellar environments with Lyman absorption by the neutral intergalactic medium. We find that JWST and WFIRST will detect these explosions out to $z \sim 30$ and 20, respectively, unveiling the first generation of stars in the universe.
\end{abstract}

Subject headings: early universe - galaxies: high-redshift - stars: early-type - supernovae: general radiative transfer - hydrodynamics - shocks

\section{INTRODUCTION}

The cosmic Dark Ages ended with the formation of the first stars in $10^{5}-10^{6} \mathrm{M}_{\odot}$ cosmological halos at $z \sim 20-30$. Besides ionizing the universe with intense UV flux and forming the first heavy elements, Pop III stars also determined the luminosities and spectra of primeval galaxies and may have been the origin of the supermassive black holes (SMBHs) at the centers of most massive galaxies today. Unfortunately, because they lie at the edge of the observable universe, individual Pop III stars will likely remain beyond the reach of direct observation for decades to come despite their enormous luminosities (Schaerer 2002) and new near infrared (NIR) observatories such as JWST (Gardner et al. 2006) and the Thirty Meter Telescope (TMT). Past numerical simulations have suggested that Pop III stars form in isolation in halos, with masses of 30-300 M $\odot$ (Nakamura \& Umemura 2001; Bromm et al. 2002; Abel et al. 2002; O'Shea \& Norman 2007), but more recent calculations point to the possibility of binaries (Turk et al. 2009), and perhaps small swarms of less massive 20-40 M. stars (Stacv et al. 2010; Clark et al. 2011; Greif et al. 2011; Hosokawa et al. 2011; Greif et al. 2012). However, in spite of their increasing sophistication numerical simulations cannot yet constrain the proper-

\footnotetext{
${ }^{1}$ McWilliams Fellow, Department of Physics, Carnegie Mellon University, Pittsburgh, PA 15213

2 CCS-2, Los Alamos National Laboratory, Los Alamos, NM 87545

${ }^{3}$ Enrico Fermi Institute, Department of Physics, and Kavli Institute for Cosmological Physics, University of Chicago, Chicago, IL 60637, US

${ }^{4}$ Monash Centre for Astrophysics, Monash University, Victoria, 3800, Australia

${ }^{5}$ Department of Astronomy and Astrophysics, UCSC, Santa Cruz, CA 95064

6 Space Telescope Science Institute, 3700 San Martin Drive, Baltimore, MD 21218

7 XTD-6, Los Alamos National Laboratory, Los Alamos, NM 87545

8 Department of Computer Science, University of New Mexico, Albuquerque, NM 87131
}

ties of the first stars, as they do not realistically bridge the gap between the formation and fragmentation of protostellar disks and their destruction by nascent stars up to a Myr later.

It may soon be possible to deduce the properties of Pop III stars from their supernova (SN) explosions. Primordial stars from 10-40 $\mathrm{M}_{\odot}$ are thought to die in core-collapse SNe while 140-260 $\mathrm{M}_{\odot}$ stars explode as pair-instability (PI) SNe, extremely energetic thermonuclear explosions with up to 100 times the energy of Type Ia or II SNe (Heger \& Wooslev 2002; Whalen et al. 2008b; Joggerst et al. 2010; Heger \& Wooslev 2010; Joggerst \& Whalen 2011). Pop III stars above $100 \mathrm{M}_{\odot}$ encounter the pair instability after central carbon burning, when thermal energy creates electron-positron pairs rather than providing pressure support against collapse (Barkat et al. 1967). Their cores subsequently contract, triggering explosive thermonuclear burning of $\mathrm{O}$ and $\mathrm{Si}$. Above $140 \mathrm{M}_{\odot}$ the energy released completely unbinds the star, with no compact remnant. At $260 \mathrm{M}_{\odot}$ the core becomes so hot that alpha particles are photodisintegrated into free nucleons, consuming as much energy per unit mass as was released by all preceding burning, and the star collapses instead of exploding. PI SNe such as SN 2007bi have now been discovered in the local universe (Gal-Yam et al. 2009), and the recent discovery that rotating Pop III stars can die as PI SNe at masses as low as $65 \mathrm{M}_{\odot}$ may increase the number of such explosions at high redshifts by a factor of a few (Chatzopoulos \& Wheeler 2012).

Pop III PI SNe could be ideal probes of the primeval universe because they are 100,000 times brighter than their progenitors and the early galaxies in which they reside, and might be found by $J W S T$ or in all-sky surveys by WFIRST or the Wide-field Imaging Surveyor for High-Redshift (WISH). However, to determine if these events can be seen from Earth, fully radiationhydrodynamical simulations of PI SNe must be performed in realistic circumstellar envelopes, whose opacity and interaction with the shock crucially shape the 
TABLE 1

Pop III PI SN properties (MASses ARE IN $\mathrm{M}_{\odot}$ )

\begin{tabular}{ccccc}
\hline \hline $\mathrm{M}_{\star}$ & $M_{\mathrm{He}}$ & $R\left(10^{14} \mathrm{~cm}\right)$ & $E\left(10^{51} \mathrm{erg}\right)$ & $M_{56} \mathrm{Ni}$ \\
\hline 150 & 72 & 1.62 & 9.0 & 0.07 \\
175 & 84.4 & 1.74 & 21.3 & 0.70 \\
200 & 96.7 & 1.84 & 33 & 5.09 \\
225 & 103.5 & 3.33 & 46.7 & 16.5 \\
250 & 124 & 2.25 & 69.2 & 37.9 \\
\hline
\end{tabular}

temperatures and spectra in the source frame, and thus the flux that is eventually redshifted into the NIR. Lyman absorption by neutral hydrogen prior to the era of reionization, which can absorb or scatter light from these ancient supernovae out of our line of sight, must also be taken into account for explosions at the earliest epochs. Previous simulations of PI SNe have not included these effects (Scannapieco et al. 2005; Kasen et al. 2011), and estimates of the redshift at which they can be detected have either been confined to $z<15$ (the era of primitive galaxies rather than the epoch of first light) or have been attempted at $z \sim 30$ with only very approximate methods (Pan et al. 2012a, b; Hummel et al. 2012).

We have performed numerical simulations of the NIR light curves of Pop III PI SNe. In $\oint 2$ we describe our numerical method and in $\S 3$ we present source frame light curves and spectra for 5 PI explosions. In $\S 4$ NIR light curves for these events are shown for several redshifts, and we discuss their detection limits in redshift.

\section{NUMERICAL METHOD}

Our calculations are done in four stages. First, we model the evolution and initial explosion of 150, 175, 200, 225, and $250 \mathrm{M}_{\odot}$ Pop III stars in the Kepler code (Weaver et al. 1978; Wooslev et al. 2002). Second, the shock is propagated through the interior of the star, its surface, and then out into the surrounding medium with the radiation hydrodynamics code RAGE (Gittings et al. 2008). We model radiation flows with grey flux-limited diffusion and evolve matter and radiation temperatures separately because they are not always in equilibrium. This physics enables us to better capture shock breakout (e.g. Matzner \& McKee 1999; Nakar \& Sari 2010; Piro et al. 2010; Katz et al.|2012), as discussed in greater detail in (Frey et al. 2012). Energy deposition in the gas due to the radioactive decay of ${ }^{56} \mathrm{Ni}$, which powers the light curve at later times, is also included.

We surround the star with a power-law wind profile with a total mass of $0.1 \mathrm{M}_{\odot}$, in keeping with the general consensus that massive zero-metallicity stars do not exhibit much mass loss due to their pristine atmospheres (Vink et al. 2001). The wind profile is smoothly joined to the H II region density profile of a massive Pop III star calculated with the ZEUS-MP code (Whalen et al. 2004; Whalen \& Norman 2006). ZEUS-MP also captures the ionization state of the wind due to UV radiation from the star, and therefore its opacity to radiation from the SN. Because all five stars become red hypergiants and cease to emit ionizing radiation in the final few hundred kyr of their lives, the wind completely recombines by the time they explode while the low-density H II region beyond it remains ionized.

Third, we construct spectra from the RAGE blast profiles with the SPECTRUM code (Frey et al. 2012) using

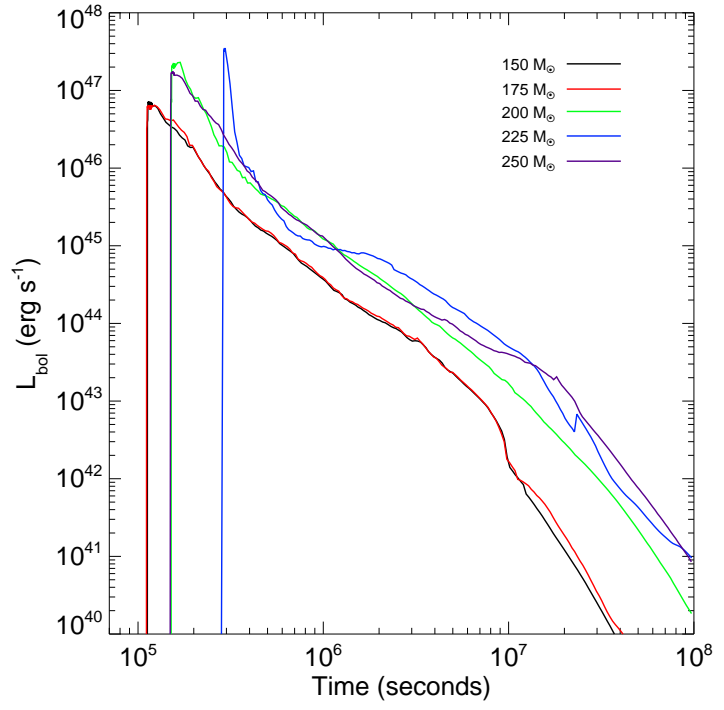

FIG. 1.- Source frame bolometric luminosities for the 5 PI SNe. The general trend of higher luminosity with progenitor mass is due to the larger explosion energies and mass of ${ }^{56} \mathrm{Ni}$ that is synthesized.

atomic opacities from the Los Alamos OPLIB database (Magee et al. 1995). SPECTRUM calculates continuum and line luminosities, absorption lines imprinted on the flux by the ejecta and envelope, and Doppler shifts and time dilation due to relativistic expansion. Finally, we convolve our spectra with Lyman absorption by neutral clouds (Madau 1995; Su et al. 2011), cosmological redshifting, and filter response functions to calculate NIR light curves. We summarize the properties of our explosions in Table 1.

\section{SOURCE FRAME LIGHT CURVES / SPECTRA}

Bolometric source frame luminosities are shown for all five explosions in Figure [1. The shock is not visible to an external observer before breaking through the surface of the star because electrons in the intervening layers scatter the photons. When it reaches the surface, the shock abruptly accelerates in the steep density gradient, becoming even hotter and releasing a brilliant pulse of photons with peak luminosities above $10^{47} \mathrm{erg} \mathrm{s}^{-1}$, or 2500 times the luminosity of the Milky Way galaxy. The transient is mostly X-rays and hard UV, and its duration is related to the radius of the star, since photons emitted simultaneously from its poles and equator reach an observer at times that are separated by the time it takes light to cross the star. The temperature of the shock at breakout is $\sim 10^{6} \mathrm{~K}$. After the initial transient subsides, the luminosities gradually decline from $10^{46} \mathrm{erg} \mathrm{s}^{-1}$ to $10^{42} \mathrm{erg} \mathrm{s}^{-1}$ over $3 \mathrm{yr}$ as gamma rays from the decay of ${ }^{56} \mathrm{Ni}$ formed during the explosion downscatter in energy and diffuse out through the ejecta.

Pair-instability SN light curves are powered at early times by the conversion of kinetic energy into thermal energy by the shock, and are thus far more luminous than Type Ia and II SNe because they have much higher explosion energies. At later times they are primarily powered by radioactive decay, and they remain much brighter than other SNe because they have synthesized more ${ }^{56} \mathrm{Ni}$ : up to $50 \mathrm{M}_{\odot}$ compared to $0.4-0.8 \mathrm{M}_{\odot}$ and $<0.3 \mathrm{M}_{\odot}$ in Type Ia and II SNe, respectively. They are luminous for 


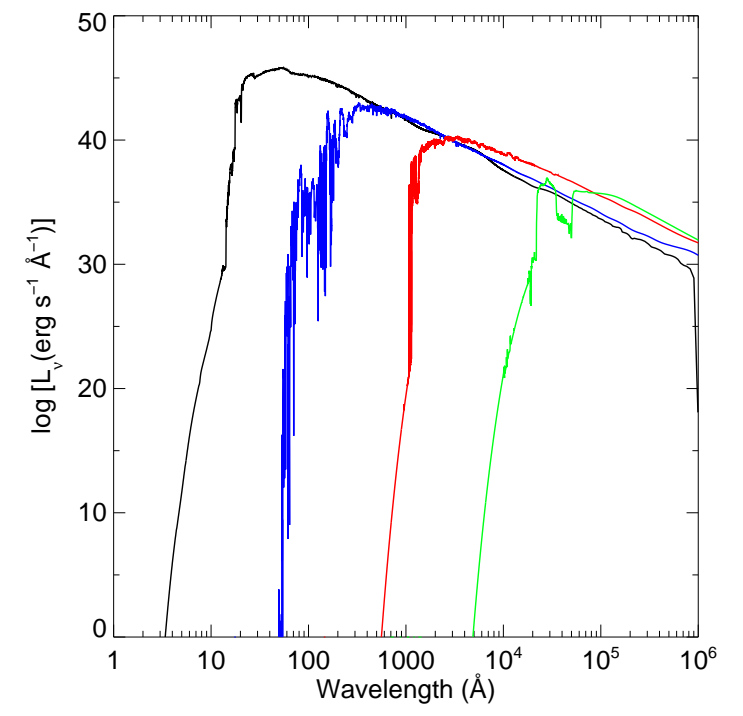

FIG. 2.- Spectral evolution of the $225 \mathrm{M}_{\odot}$ pair instability supernova. The fireball spectra are for $2.91 \times 10^{5} \mathrm{~s}$ (3.4 days, black), $6.13 \times 10^{5} \mathrm{~s}(7$ days, blue $), 9.74 \times 10^{6} \mathrm{~s}$ (113 days, red $)$ and 9.70 $\times 10^{7} \mathrm{~s}$ (1123 days, green).

longer times (3 yr instead of 3-6 mo for Type Ia and II $\mathrm{SNe}$ ) because radiation diffusion timescales in their more massive ejecta are longer:

$$
t_{d} \sim \kappa^{\frac{1}{2}} M_{\mathrm{ej}}^{\frac{3}{4}} E^{-\frac{3}{4}} .
$$

Here, $\kappa$ is the average opacity of the ejecta, $\mathrm{M}_{\mathrm{ej}}$ is the mass of the ejecta, and $E$ is the explosion energy. All five light curves slightly rebrighten at 4 months when the photosphere (the surface from which photons stream freely into space) recedes into the hot ${ }^{56} \mathrm{Ni}$ layer deep in the ejecta. This emission peaks at $\sim 3000 \AA$, and its luminosity increases with the mass of ${ }^{56} \mathrm{Ni}$, and hence the mass of the progenitor. Rebrightening occurs later in more massive stars because more time is required for the photosphere to sink into the ${ }^{56} \mathrm{Ni}$ in the frame of the shock.

Figure 2 shows spectra for the $225 \mathrm{M}_{\odot}$ explosion at four times ranging from shock breakout to $3 \mathrm{yr}$. As the fireball expands it cools, and its spectral peak advances to longer wavelengths. The breakout transient ionizes the envelope shrouding the star, as seen at $2.9 \times 10^{5} \mathrm{~s}$ (3.4 days) when the spectrum has far fewer absorption lines than at later times when the shock cools and can no longer fully ionize the wind. As the envelope recombines it imprints many more absorption lines on the spectrum, especially at shorter wavelengths where line blanketing effectively shears off the spectrum. Consequently, the common practice of fitting blackbodies to bolometric light curves to approximate shock spectra can seriously overestimate the NIR flux in the observer frame, because such profiles have a significant amount of flux at short wavelengths that is actually removed by the envelope and ejecta. On the other hand, our bolometric luminosities are an order of magnitude higher at shock breakout than previous PI SN light curves because the shock crashes into the wind and heats it, and because radiation and matter are not forced to be in thermal equilibrium in our calculations. By 3 yr most of the photons in the

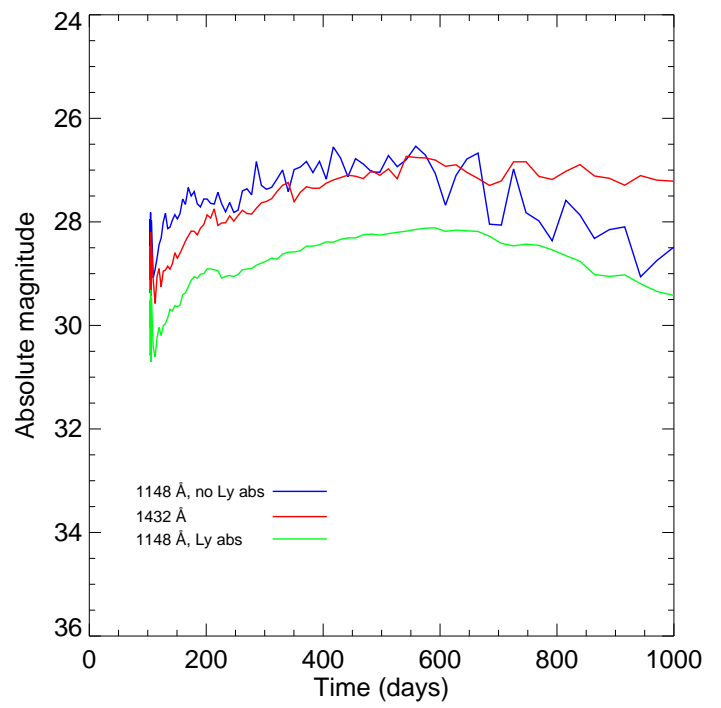

FIG. 3.- NIR light curves with and without Lyman absorption at $z=30$. Blue: $3.56 \mu \mathrm{m}$ (1148 $\AA$ in the source frame), no absorption. Green: $3.56 \mu \mathrm{m}$ with absorption. Red: $4.44 \mu \mathrm{m}$ (1432 $\AA$ in the source frame).

spectrum are below the ionization limit of hydrogen and pass through the envelope, although resonant scattering continues to imprint absorption lines on flux below this limit.

\section{JWST AND WFIRST DETECTION LIMITS}

At high redshift, Lyman absorption will absorb most luminosity at wavelengths far blueward of $1216 \AA$. However, it could be possible that the fireball is brighter a little blueward of the Lyman limit rather than a little redward, even with absorption. To determine in which $J W S T$ NIRCam filter an explosion is brightest at a given redshift, we calculate its NIR signal at wavelengths above and below the Lyman limit in the observer frame. At $z=$ 30 we find that although all five SNe are brighter just below $1216 \AA$ in the source frame, IGM absorption reduces these luminosities to magnitudes below those just redward of the limit for which there is no absorption, as we show for the $225 \mathrm{M}_{\odot}$ PI SN in Figure 3 , The explosion is brighter at $1148 \AA$ in the source frame $(3.56 \mu \mathrm{m}$ in the observer frame) at early times than at $1432 \AA$ (4.44 $\mu \mathrm{m}$ in the observer frame), but after IGM absorption is taken into account the event is brighter at $4.44 \mu \mathrm{m}$ in the observer frame.

In the left panel of Figure 4 we show NIR light curves for all five Pop III SNe at $4.44 \mu \mathrm{m}$ in JWST at $z=30$, or $100 \mathrm{Myr}$ after the big bang. The detection limit for this filter is AB magnitude 32, so all five explosions will be visible for over a thousand days. Their light curves do not cross above the detection threshold until 50 days (or 1.5 days in the source frame at this redshift), so the most luminous component of the explosion, shock breakout, cannot be seen from Earth. The X-rays and extreme UV in the pulse are all redshifted into the UV and absorbed by neutral hydrogen.

The NIR flux in the observer frame exhibits significantly more variability than the bolometric flux because of the expansion and cooling of the fireball, and this is essential to identifying these events as explosive tran- 

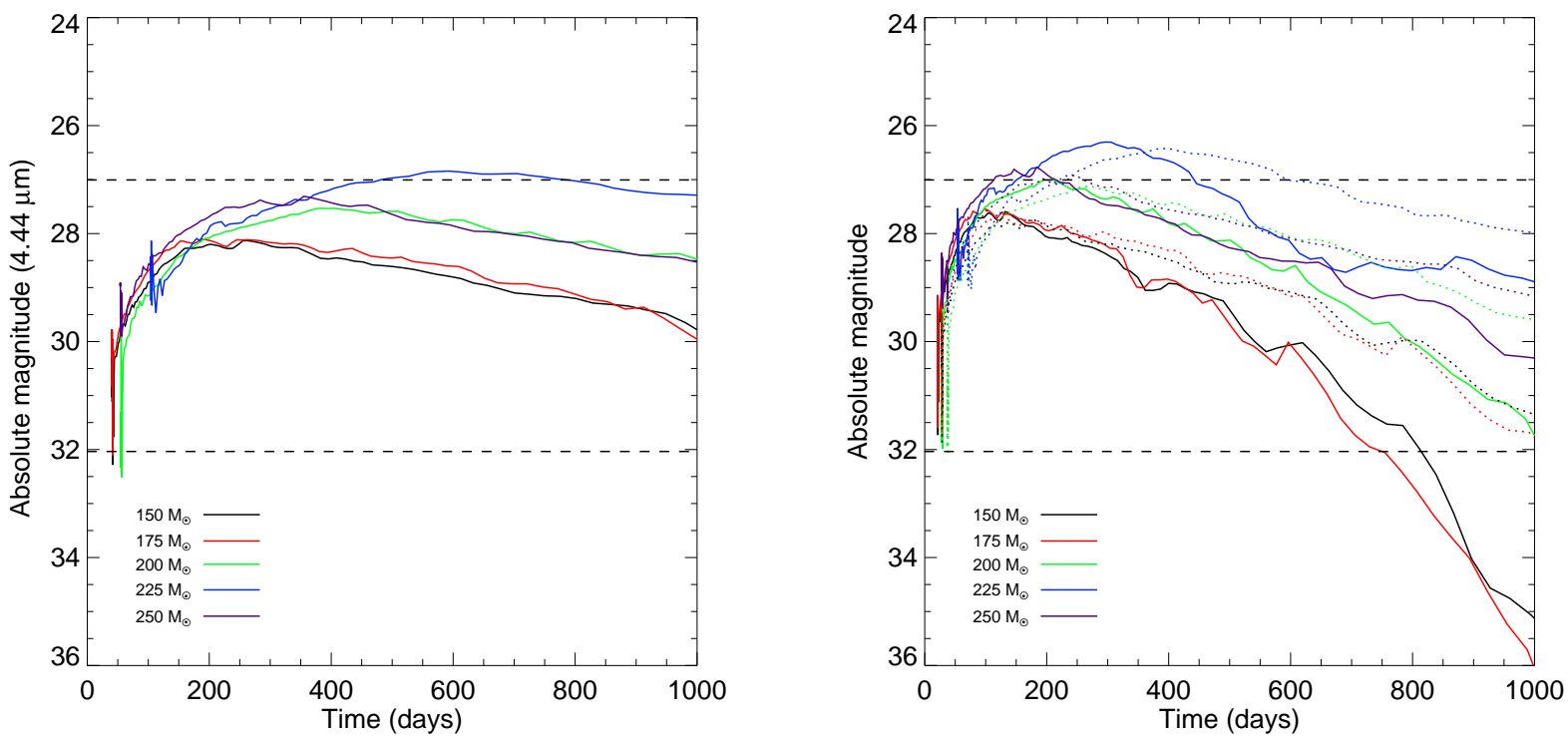

FIg. 4. - NIR light curves for five Pop III PI SNe. Left: NIR magnitudes at $4.44 \mu \mathrm{m}$ at $z=30,100$ Myr after the big bang. The horizontal dashed lines at mag 32 and 27 are JWST and WFIRST detection limits, respectively. The explosions remain above the NIRCam detection limit for over 1,000 days. Right: NIR light curves at $2.0 \mu \mathrm{m}$ at $z=15$ (solid) and $z=20$ (dotted).

sients. The flux rises much more quickly than it falls, so it is easiest to detect Pop III PI SNe in their initial stages. Nonetheless, they exhibit sufficient variability over likely protogalactic survey times of $1-5$ yr to be identified at later times as well. This variation over timescales of $\sim 1000$ days will also allow them to be discriminated from primeval galaxies, in spite of their probable overlap in color-color space. Although recent calculations show that roughly one Pop III PI SN will be present in any given $J W S T$ deep field (Trenti et al. 2009; Hummel et al. 2012; Johnson et al. 2012), far greater numbers could be found in proposed all-sky NIR surveys with WFIRST. With a sensitivity of AB magnitude 27 at $2.2 \mu \mathrm{m}$, WFIRST will see such explosions at $z=15-20$, as we show in the right panel of Figure 4. Such redshifts may be optimal for detecting PI SNe because of the rise of UV background fields from the first generations of Pop III stars. This background is thought to delay primordial star formation in halos until they have grown to larger masses, forming more massive stars at slightly lower redshifts (Wise \& Abel 2007; O'Shea \& Norman 2008). Star formation rates extracted from large-scale cosmological simulations of early structure formation that include UV backgrounds (Johnson et al. 2012) suggest that WFIRST will detect up to $10^{3}$ PI SNe per year at $15<z<20$. Detections of this volume will enable the first major surveys of the Pop III initial mass function (IMF) (see Tanaka et al. 2012; Moriva et al. 2012, for discussions on strategies for detecting superluminous Type IIn SNe in all-sky surveys).

Our calculations definitively establish that JWST and
WFIRST will be able to detect Pop III supernovae at the era of first light. In addition to unveiling the nature of primordial stars and constraining scenarios for early cosmological reionization and chemical enrichment, detections of Pop III supernovae at slightly lower redshifts $(z \sim 10-15)$ will probe the era of primitive galaxy formation, revealing their positions on the sky when they might otherwise have eluded detection by JWST. The discovery of the first cosmic explosions will be one of the most spectacular results in extragalactic astronomy in the coming decade, and open the first window on the end of the cosmic Dark Ages.

The authors thank the anonymous referee, whose comments improved the quality of this paper. DJW was supported by the Bruce and Astrid McWilliams Center for Cosmology at Carnegie Mellon University. DEH acknowledges support from the National Science Foundation CAREER grant PHY-1151836. AH was supported by the US Department of Energy under contracts DEFC02-01ER41176, FC02-09ER41618 (SciDAC), and DEFG02-87ER40328. SEW was supported by the National Science Foundation grant AST 0909129 and the NASA Theory Program grant NNX09AK36G. MS thanks Marcia Rieke for making available the NIRCam filter curves, and was partially supported by NASA JWST grant NAG5-12458. Work at LANL was done under the auspices of the National Nuclear Security Administration of the U.S. Department of Energy at Los Alamos National Laboratory under Contract No. DE-AC52-06NA25396.

\section{REFERENCES}

Abel, T., Bryan, G. L., \& Norman, M. L. 2002, Science, 295, 93

Barkat, Z., Rakavy, G., \& Sack, N. 1967, Physical Review Letters, 18, 379

Bromm, V., Coppi, P. S., \& Larson, R. B. 2002, ApJ, 564, 23

Chatzopoulos, E. \& Wheeler, J. C. 2012, ApJ, 748, 42

Clark, P. C., Glover, S. C. O., Smith, R. J., Greif, T. H., Klessen, R. S., \& Bromm, V. 2011, Science, 331, 1040
Frey, L. H., Even, W., Whalen, D. J., Fryer, C. L., Hungerford, A. L., Fontes, C. J., \& Colgan, J. 2012, arXiv:1203.5832 
Gal-Yam, A., Mazzali, P., Ofek, E. O., Nugent, P. E., Kulkarni, S. R., Kasliwal, M. M., Quimby, R. M., Filippenko, A. V. Cenko, S. B., Chornock, R., Waldman, R., Kasen, D., Sullivan, M., Beshore, E. C., Drake, A. J., Thomas, R. C., Bloom, J. S., Poznanski, D., Miller, A. A., Foley, R. J., Silverman, J. M., Arcavi, I., Ellis, R. S., \& Deng, J. 2009, Nature, 462, 624

Gardner, J. P., Mather, J. C., Clampin, M., Doyon, R., Greenhouse, M. A., Hammel, H. B., Hutchings, J. B., Jakobsen, P., Lilly, S. J., Long, K. S., Lunine, J. I., McCaughrean, M. J., Mountain, M., Nella, J., Rieke, G. H., Rieke, M. J., Rix, H.-W., Smith, E. P., Sonneborn, G., Stiavelli, M., Stockman, H. S., Windhorst, R. A., \& Wright, G. S. 2006, Space Sci. Rev., 123, 485

Gittings, M., Weaver, R., Clover, M., Betlach, T., Byrne, N. Coker, R., Dendy, E., Hueckstaedt, R., New, K., Oakes, W. R., Ranta, D., \& Stefan, R. 2008, Computational Science and Discovery, 1, 015005

Greif, T. H., Bromm, V., Clark, P. C., Glover, S. C. O., Smith, R. J., Klessen, R. S., Yoshida, N., \& Springel, V. 2012, MNRAS, 424, 399

Greif, T. H., Springel, V., White, S. D. M., Glover, S. C. O., Clark, P. C., Smith, R. J., Klessen, R. S., \& Bromm, V. 2011, ApJ, 737, 75

Heger, A. \& Woosley, S. E. 2002, ApJ, 567, 532

-. 2010, ApJ, 724, 341

Hosokawa, T., Omukai, K., Yoshida, N., \& Yorke, H. W. 2011, Science, 334, 1250

Hummel, J. A., Pawlik, A. H., Milosavljević, M., \& Bromm, V. 2012, ApJ, 755, 72

Joggerst, C. C., Almgren, A., Bell, J., Heger, A., Whalen, D., \& Woosley, S. E. 2010, ApJ, 709, 11

Joggerst, C. C. \& Whalen, D. J. 2011, ApJ, 728, 129

Johnson, J. L., Dalla Vecchia, C., \& Khochfar, S. 2012, arXiv: 1206.5824

Kasen, D., Woosley, S. E., \& Heger, A. 2011, ApJ, 734, 102

Katz, B., Sapir, N., \& Waxman, E. 2012, ApJ, 747, 147

Madau, P. 1995, ApJ, 441, 18

Magee, N. H., Abdallah, Jr., J., Clark, R. E. H., Cohen, J. S., Collins, L. A., Csanak, G., Fontes, C. J., Gauger, A., Keady, J. J., Kilcrease, D. P., \& Merts, A. L. 1995, in Astronomical Society of the Pacific Conference Series, Vol. 78, Astrophysical Applications of Powerful New Databases, ed. S. J. Adelman \& W. L. Wiese, 51
Matzner, C. D. \& McKee, C. F. 1999, ApJ, 510, 379

Moriya, T. J., Blinnikov, S. I., Tominaga, N., Yoshida, N., Tanaka, M., Maeda, K., \& Nomoto, K. 2012, arXiv:1204.6109

Nakamura, F. \& Umemura, M. 2001, ApJ, 548, 19

Nakar, E. \& Sari, R. 2010, ApJ, 725, 904

O'Shea, B. W. \& Norman, M. L. 2007, ApJ, 654, 66

—. 2008, ApJ, 673, 14

Pan, T., Kasen, D., \& Loeb, A. 2012a, MNRAS, 422, 2701

Pan, T., Loeb, A., \& Kasen, D. 2012b, MNRAS, 423, 2203

Piro, A. L., Chang, P., \& Weinberg, N. N. 2010, ApJ, 708, 598

Scannapieco, E., Madau, P., Woosley, S., Heger, A., \& Ferrara, A. 2005, ApJ, 633, 1031

Schaerer, D. 2002, A\&A, 382, 28

Stacy, A., Greif, T. H., \& Bromm, V. 2010, MNRAS, 403, 45

Su, J., Stiavelli, M., Oesch, P., Trenti, M., Bergeron, E., Bradley, L., Carollo, M., Dahlen, T., Ferguson, H. C., Giavalisco, M., Koekemoer, A., Lilly, S., Lucas, R. A., Mobasher, B., Panagia, N., \& Pavlovsky, C. 2011, ApJ, 738, 123

Tanaka, M., Moriya, T. J., Yoshida, N., \& Nomoto, K. 2012, MNRAS, 422, 2675

Trenti, M., Stiavelli, M., \& Michael Shull, J. 2009, ApJ, 700, 1672

Turk, M. J., Abel, T., \& O'Shea, B. 2009, Science, 325, 601

Vink, J. S., de Koter, A., \& Lamers, H. J. G. L. M. 2001, A\&A, 369,574

Weaver, T. A., Zimmerman, G. B., \& Woosley, S. E. 1978, ApJ, 225,1021

Whalen, D., Abel, T., \& Norman, M. L. 2004, ApJ, 610, 14

Whalen, D. \& Norman, M. L. 2006, ApJS, 162, 281

Whalen, D., van Veelen, B., O'Shea, B. W., \& Norman, M. L. 2008b, ApJ, 682, 49

Wise, J. H. \& Abel, T. 2007, ApJ, 671, 1559

Woosley, S. E., Heger, A., \& Weaver, T. A. 2002, Reviews of Modern Physics, 74, 1015 